\title{
A Rate-Distortion Theory for Permutation Spaces
}

\author{
Da Wang \\ EECS Dept., MIT \\ Cambridge, MA, USA \\ Email: dawang@mit . edu
}

\author{
Arya Mazumdar \\ ECE Dept., University of Minnesota \\ Twin Cities, MN, USA \\ Email: arya@umn . edu
}

\author{
Gregory W. Wornell \\ EECS Dept., MIT \\ Cambridge, MA, USA \\ Email: gww@mit . edu
}

\begin{abstract}
We investigate the lossy compression of the permutation space by analyzing the trade-off between the size of a source code and the distortion with respect to either Kendall tau distance or $\ell_{1}$ distance of the inversion vectors. For both distortion measures, we characterize the rate-distortion functions and provide explicit code designs that achieve them. Finally, we provide bounds on the higher order terms in the codebook size when the distortion levels lead to degenerate code rates $(0$ or 1$)$.
\end{abstract}

\section{INTRODUCTION}

Permutations are fundamental mathematical objects and codes in permutations is a classical subject in coding theory, with a variety of applications that correspond to different metric functions on the symmetric group $\mathcal{S}_{n}$. For example, some works focus on codes in $\mathcal{S}_{n}$ with Hamming distance [1], [2], and some others investigate the error correction problem under metrics such as Chebyshev distance [3] and Kendall tau distance [4].

While error correction problems in permutation spaces have been investigated, the lossy compression problem is largely left unattended. In a series of work, [5], [6], [7] investigate the lossless compression of a group of permutations with certain properties, such as efficient rank querying (given an element, get its rank in the permutation) and selection (given a rank, retrieve the corresponding element). In addition to compression, lossy source coding of permutations is related to the problem of comparisonbased approximate sorting, which can be seen as searching a true permutation subject to certain distortion. Since each comparison in sorting provides at most 1 bit of information (assuming all elements are distinct), the code size provides a lower bound to the required number of comparisons. The problem of approximate sorting has been investigated in [8], where results for the moderate distortion regime (see below for definition) are derived with respect to the Spearman's footrule metric [9].

In this paper we investigate the lossy compression of $\mathcal{S}_{n}$, with distortion measure being either the Kendall tau distance and $\ell_{1}$ distance of the inversion vectors. Following the classical rate distortion setup, we analyze the required rate $R$ to achieve worst-case distortion $D$. We show that for the distortion $D=\Theta\left(n^{1+\delta}\right),-1 \leq \delta \leq 1$, $R=1-\max \{\delta, 0\}$. Furthermore, for the small (sublinear and linear) distortion regime $(-1<\delta \leq 0)$ and large (quadratic) distortion regime $(\delta=1)$, we analyze the higher order terms in the required rate $R$ to provide more insights

This work was supported, in part, by AFOSR under Grant No. FA955011-1-0183, and by NSF under Grant No. CCF-1017772. Arya Mazumdar's research was also supported in part by a startup grant from University of Minnesota. on how rate changes as we vary distortion. Finally, our achievability schemes are constructive and hence provide explicit code designs.

The rest of the paper is organized as follows. We first present the problem formulation in Section II. We discuss some geometric properties of permutation spaces in Section III. Then we characterize the rate-distortion trade-offs for permutation spaces with the Kendall tau distance and the $\ell_{1}$ distance of the inversion vectors in Section IV and Section V respectively.

\section{PROBLEM FORMULATION}

In this section we provide a mathematical formulation for the rate-distortion problem on a permutation space.

We first formally define a permutation space. Let $\mathcal{S}_{n}$ denote the symmetric group of $n$ elements. We write the elements of $\mathcal{S}_{n}$ as arrays of natural numbers with values ranging from $1, \ldots, n$ and every value occurring only once in the array. Given a metric $d: \mathcal{S}_{n} \times \mathcal{S}_{n} \rightarrow \mathbb{R}^{+} \cup\{0\}$, we define a permutation space $\mathcal{X}\left(\mathcal{S}_{n}, d\right)$.

In this paper, we denote the set $\{1, \ldots, n\}$ as $[n]$, and let $[a: b] \triangleq\{a, a+1, \ldots, b-1, b\}$ for any two integers $a$ and $b$.

The notions of inversion and inversion vector play important roles through the course of this paper.

Definition 1 (inversion, inversion vector). An inversion in a permutation $\sigma \in \mathcal{S}_{n}$ is a pair $(\sigma(i), \sigma(j))$ such that $i<j$ and $\sigma(i)>\sigma(j)$. We use $I_{n}(\sigma)$ to denote the total number of inversions in $\sigma \in \mathcal{S}_{n}$, and

$$
K_{n}(k) \triangleq\left|\left\{\sigma \in \mathcal{S}_{n}: I_{n}(\sigma)=k\right\}\right|
$$

to denote the number of permutations with $k$ inversions.

A permutation $\sigma \in \mathcal{S}_{n}$ is associated with an inversion vector $\mathbf{x}_{\sigma}$ in the set of inversion vectors $\mathcal{G}_{n} \triangleq[0: 1] \times[0$ : $2] \times \cdots \times[0: n-1]$, where for $i=1, \ldots, n-1$,

$$
\mathbf{x}_{\sigma}(i)=\left|\left\{j \in[n]: j<i+1, \sigma^{-1}(j)>\sigma^{-1}(i+1)\right\}\right| .
$$

In words, $\mathbf{x}_{\sigma}(i)$ is the number of inversions in $\sigma$ in which $i+1$ is the first element.

It is well-known that mapping from $\mathcal{S}_{n}$ to $\mathcal{G}_{n}$ is oneto-one and straightforward [10]. As an example, $\sigma=$ $[2,1,6,4,3,7,5,9,8]$ corresponds to the inversion vector $\mathbf{x}_{\sigma}=[1,0,1,0,3,1,0,1]$.

Definition 2 (Kendall tau distance). The Kendall tau distance $d_{\tau}\left(\sigma_{1}, \sigma_{2}\right)$ from one permutation $\sigma_{1}$ to another permutation $\sigma_{2}$ is defined as the minimum number of transpositions of pairwise adjacent elements required to change $\sigma_{1}$ into $\sigma_{2}$. Note that the Kendall tau distance is upper bounded by $\left(\begin{array}{l}n \\ 2\end{array}\right)$. 
The Kendall tau distance is closely related to the $\ell_{1}$ distance on the $\mathcal{S}_{n}$. [9] shows that

$$
d_{\ell_{1}}\left(\sigma_{1}, \sigma_{2}\right) / 2 \leq d_{\tau}\left(\sigma_{1}^{-1}, \sigma_{2}^{-1}\right) \leq d_{\ell_{1}}\left(\sigma_{1}, \sigma_{2}\right),
$$

where $\sigma^{-1}$ is the permutation inverse of $\sigma$ and $d_{\ell_{1}}(\cdot, \cdot)$ is the $\ell_{1}$ distance, $d_{\ell_{1}}\left(\sigma_{1}, \sigma_{2}\right) \triangleq \sum_{i=1}^{n}\left|\sigma_{1}(i)-\sigma_{2}(i)\right|$.

In addition, the Kendall tau distance between two permutation vectors provides an upper bound to the $\ell_{1}$ distance between the inversion vectors of the corresponding permutations [11], i.e., given two permutations $\sigma_{1}$ and $\sigma_{2}$,

$$
d_{\mathbf{x}, \ell_{1}}\left(\sigma_{1}, \sigma_{2}\right) \leq d_{\tau}\left(\sigma_{1}, \sigma_{2}\right)
$$

where $d_{\mathbf{x}, \ell_{1}}\left(\sigma_{1}, \sigma_{2}\right) \triangleq \sum_{i=1}^{n-1}\left|\mathbf{x}_{\sigma_{1}}(i)-\mathbf{x}_{\sigma_{2}}(i)\right|$.

Now we define the rate-distortion problem on a permutation space.

Definition 3 (codebook for permutations). A ( $\left.n, D_{n}\right)$ source code $\mathcal{C}_{n} \subset \mathcal{X}\left(\mathcal{S}_{n}, d\right)$ is a set of $M_{n}$ permutations such that for any $\sigma \in \mathcal{S}_{n}$, there exists $\pi \in \mathcal{C}_{n}$ that $d(\pi, \sigma) \leq D_{n}$. Let $A\left(n, D_{n}\right)$ be minimum size of the $\left(n, D_{n}\right)$ source codes in $\mathcal{X}\left(\mathcal{S}_{n}, d\right)$ with distortion $D_{n}$. We define the minimal rate for distortion $D_{n}$ as

$$
R\left(D_{n}\right) \triangleq \frac{\log A\left(n, D_{n}\right)}{\log n !} .
$$

As to the classical rate-distortion setup, we are interested in deriving the trade-off between distortion level $D_{n}$ and the rate $R\left(D_{n}\right)$ as $n$ goes to $\infty$. In this work we show that $\lim _{n \rightarrow \infty} R\left(D_{n}\right)$ exists.

A close observation guarantees that when $D_{n}=O(n)$ or $D_{n}=\Omega\left(n^{2}\right), \lim _{n \rightarrow \infty} R\left(D_{n}\right)=1$ and $\lim _{n \rightarrow \infty} R\left(D_{n}\right)=$ 0 respectively. In these two scenarios, in addition to $\lim _{n \rightarrow \infty} R\left(D_{n}\right)$, the trade-off between rate and distortion is shown in the higher order terms in $\log A\left(n, D_{n}\right)$, which we investigate via the following:

$$
r\left(D_{n}\right) \triangleq \log A\left(n, D_{n}\right)-\log n ! \lim _{n \rightarrow \infty} R\left(D_{n}\right) .
$$

Remark 1. In the small distortion region with $R\left(D_{n}\right)=1$, $r\left(D_{n}\right)$ is negative while in the large distortion region where $R\left(D_{n}\right)=0, r\left(D_{n}\right)$ is positive.

Remark 2. In this paper we consider the classical combinatorial approach of worst-case distortion, while in the usual rate-distortion setup one considers the average-case distortion (w.r.t. a certain distribution of the source). However, for the uniform distribution on $\mathcal{S}_{n}$, the rate-distortion function is the same for both average- and worst-case, apart from the terms that are asymptotically negligible. In particular, our achievability schemes still hold, and the converse can be proved by showing that for any $0<\tau<1-\delta, n !^{1-\delta-\tau}$ $2 D$-balls covers less than half of the permutations in $\mathcal{S}_{n}$ and hence leads to an average distortion larger than $D$. Therefore, at least $n !^{1-\delta}$ number of $D$-balls are required for average distortion to be no larger than $D$.

However, the higher order term $r(D)$ does behave differently under average and worst-case distortion.

Finally, we define Stirling's approximation, a frequentlyused technique in this paper.
Definition 4 (Stirling's approximation). For $m \in \mathbb{Z}^{+}$,

$$
\sqrt{2 \pi m}\left(\frac{m}{e}\right)^{m} e^{\frac{1}{12 m+1}}<m !<\sqrt{2 \pi m}\left(\frac{m}{e}\right)^{m} e^{\frac{1}{12 m}} .
$$

\section{GeOMETRY OF PERMUTATION SPACES}

In this section we provide results on the geometry of the permutation space that are useful in deriving the ratedistortion bounds.

We first define $D$-balls centered at $\sigma \in \mathcal{S}_{n}$ with radius $D$ under distance $d(\cdot, \cdot)$ and their maximum sizes:

$$
\begin{aligned}
B_{d}(\sigma, D) & \triangleq\{\pi: d(\pi, \sigma) \leq D\}, \\
N_{d}(D) & \triangleq \max _{\sigma \in \mathcal{S}_{n}}\left|B_{d}(\sigma, D)\right| .
\end{aligned}
$$

Let $B_{\tau}(\sigma, D), B_{\ell_{1}}(\sigma, D)$ and $B_{\mathbf{x}, \ell_{1}}(\sigma, D)$ be the balls that correspond to the Kendall tau distance, $\ell_{1}$ distance of the permutations, and $\ell_{1}$ distance of the inversion vectors, and $N_{\tau}(D), N_{\ell_{1}}(D)$, and $N_{\mathbf{x}, \ell_{1}}(D)$ be their maximum sizes respectively.

Note that (3) implies $B_{\tau}(\sigma, D) \subset B_{\mathbf{x}, \ell_{1}}(\sigma, D)$ and thus $N_{\tau}(D) \leq N_{\mathbf{x}, \ell_{1}}(D)$. Below we establish upper bounds for $N_{\mathbf{x}, \ell_{1}}(D)$ and $N_{\tau}(D)$, which are useful for establishing converse results later.

Lemma 1. For $0 \leq D \leq n$,

$$
N_{\tau}(D) \leq\left(\begin{array}{c}
n+D-1 \\
D
\end{array}\right) .
$$

Proof: Let the number of permutations in $\mathcal{S}_{n}$ with at most $k$ inversions be $T_{n}(d) \triangleq \sum_{k=0}^{d} K_{n}(k)$, where $K_{n}(k)$ is defined in (1). Since $\mathcal{X}\left(\mathcal{S}_{n}, d_{\tau}\right)$ is a regular metric space,

$$
N_{\tau}(D)=T_{n}(D) \text {, }
$$

which is noted in several references such as [10]. An expression for $K_{n}(D)$ (and thus $T_{n}(D)$ ) for $D \leq n$ appears in [10] (see [4] also). The following bound is weaker but sufficient in our context.

By induction, or [12], $T_{n}(D)=K_{n+1}(D)$ when $D \leq$ $n$. Then noting that for $k<n, K_{n}(k)=K_{n}(k-1)+$ $K_{n-1}(k)$ [10, Section 5.1.1] and for any $n \geq 2$,

$$
K_{n}(0)=1, \quad K_{n}(1)=n-1, \quad K_{n}(2)=\left(\begin{array}{l}
n \\
2
\end{array}\right)-1,
$$

by induction, we can show that when $1 \leq k<n$,

$$
K_{n}(k) \leq\left(\begin{array}{c}
n+k-2 \\
k
\end{array}\right) \text {. }
$$

The product structure of $\mathcal{X}\left(\mathcal{S}_{n}, d_{\mathbf{x}, \ell_{1}}\right)$ leads to a simpler analysis of the upper bound of $N_{\mathbf{x}, \ell_{1}}(D)$.

Lemma 2. For $0 \leq D \leq n(n-1) / 2$,

$$
N_{\mathbf{x}, \ell_{1}}(D) \leq 2^{\min \{n, D\}}\left(\begin{array}{c}
n+D \\
D
\end{array}\right) .
$$

Proof: For any $\sigma \in \mathcal{S}_{n}$, let $\mathbf{x}=\mathbf{x}_{\sigma} \in \mathcal{G}_{n}$, then $\left|B_{\mathbf{x}, \ell_{1}}(D)\right|=\sum_{r=0}^{D}\left|\left\{\mathbf{y} \in \mathcal{G}_{n}: d_{\ell_{1}}(\mathbf{x}, \mathbf{y})=r\right\}\right|$. Let $\mathbf{d} \triangleq$ $|\mathbf{x}-\mathbf{y}|$, and $Q(n, r)$ be the number of integer solutions of the equation $z_{1}+z_{2}+\ldots+z_{n}=r$ with $z_{i} \geq 0,0 \leq i \leq n$, then it is well known [13, Section 1.2] that

$$
Q(n, r)=\left(\begin{array}{c}
n+r-1 \\
r
\end{array}\right)
$$


and it is not hard to see that the number of such $\mathbf{d}=$ $\left[d_{1}, d_{2}, \ldots, d_{n-1}\right.$ that satisfies $\sum_{i=1}^{n-1} d_{i}=r$ is upper bounded by $Q(n-1, r)$. Given $\mathbf{x}$ and $\mathbf{d}$, at most $m \triangleq$ $\min \{D, n\}$ elements in $\left\{y_{i}, 0 \leq i \leq n\right\}$ correspond to $y_{i}=$ $x_{i} \pm d_{i}$. Therefore, for any $\mathbf{x},\left|\left\{\mathbf{y} \in \mathcal{G}_{n}: d_{\ell_{1}}(\mathbf{x}, \mathbf{y})=r\right\}\right| \leq$ $2^{m} Q(n, r)$ and hence

$$
\left|B_{\ell_{1}}(\mathbf{x}, D)\right| \leq \sum_{r=0}^{D} 2^{m} Q(n, r)=2^{m}\left(\begin{array}{c}
n+D \\
D
\end{array}\right)
$$

Below we upper bound $\log N_{\tau}(D)$ and $\log N_{\mathbf{x}, \ell_{1}}(D)$ for small, moderate and large $D$ regimes in Lemmas 3 to 5 respectively.

Lemma 3 (Small distortion regime). When $D=a n^{\delta}, 0<$ $\delta \leq 1$ and $a>0$ is a constant,

$$
\begin{aligned}
& \log N_{\tau}(D) \\
& \leq\left\{\begin{array}{l}
a(1-\delta) n^{\delta} \log n+O\left(n^{\delta}\right), \quad 0<\delta<1 \\
n[(1+a) \log (1+a)-a \log a]+o(n), \quad \delta=1
\end{array}\right.
\end{aligned}
$$

$$
\begin{aligned}
& \log N_{\mathbf{x}, \ell_{1}}(D) \\
& \leq\left\{\begin{array}{l}
a(1-\delta) n^{\delta} \log n+O\left(n^{\delta}\right), \quad 0<\delta<1 \\
n[2+(1+a) \log (1+a)-a \log a]+o(n), \quad \delta=1
\end{array}\right.
\end{aligned}
$$

Proof: To upper bound $N_{\tau}(D)$, when $0<\delta<1$, we apply Stirling's approximation to (7) to have

$$
\begin{aligned}
& \log \left(\begin{array}{c}
n+D-1 \\
D
\end{array}\right) \\
& =n \log \frac{n-1+D}{n-1}+D \log \frac{n-1+D}{D}+O(\log n) .
\end{aligned}
$$

Substituting $D=a n^{\delta}$, we obtain (10). When $\delta=1$, the result follows from (9) in [14, Section 4]. The upper bound on $N_{\mathbf{x}, \ell_{1}}(D)$ can be obtained similarly via (9).

Lemma 4 (Moderate distortion regime). Given $D=$ $\Theta\left(n^{1+\delta}\right), 0<\delta \leq 1$, then

$$
\log N_{\tau}(D) \leq \log N_{\mathbf{x}, \ell_{1}}(D) \leq \delta n \log n+O(n) .
$$

Proof: Apply Stirling's approximation to (9) and substitute $D=\Theta\left(n^{1+\delta}\right)$.

Remark 3. It is possible to obtain tighter lower and upper bounds for $\log N_{\tau}(D)$ and $\log N_{\mathbf{x}, \ell_{1}}(D)$ based on results in [4]. We omit the details here due to space constraints.

Lemma 5 (Large distortion regime). Given $D=b n(n-$ 1) $\in \mathbb{Z}^{+}$, then

$$
\log N_{\tau}(D) \leq \log N_{\mathbf{x}, \ell_{1}}(D) \leq n \log (2 b e n)+O(\log n) .
$$

Proof: Substitut $D=b n(n-1)$ into (9).

\section{Permutation space with Kendall tau DISTANCE}

In this section we first derive the rate-distortion function $R(D)$ for the permutation space $\mathcal{X}\left(\mathcal{S}_{n}, d_{\tau}\right)$ in the moderate distortion range. Then for small and large distortion regimes, we also characterize the higher order term $r(D)$, which is summarized in Theorem 7.

Theorem 6. In the permutation space $\mathcal{X}\left(\mathcal{S}_{n}, d_{\tau}\right)$,

$$
\lim _{n \rightarrow \infty} R\left(D_{n}\right)=\left\{\begin{array}{ll}
1 & \text { if } D=O(n) \\
1-\delta & \text { if } D=\Theta\left(n^{1+\delta}\right), \quad 0<\delta \leq 1
\end{array} .\right.
$$

Theorem 7. In the permutation space $\mathcal{X}\left(\mathcal{S}_{n}, d_{\tau}\right)$, when $D=a n^{\delta}, 0<\delta \leq 1, r_{\tau}^{\mathrm{s}}(D) \leq r(D) \leq \overline{r_{\tau}^{\mathrm{s}}(D)}$, where

$\underline{r_{\tau}^{\mathrm{s}}(D)}=\left\{\begin{array}{l}-a(1-\delta) n^{\delta} \log n+O\left(n^{\delta}\right), \quad 0<\delta<1 \\ -n[(1+a) \log (1+a)-a \log a]+o(n), \delta=1\end{array}\right.$,

$\overline{r_{\tau}^{\mathrm{s}}(D)}=\left\{\begin{array}{l}-n^{\delta} \frac{a \log 2}{2}+O(1), 0<a<1 \\ -n^{\delta} \frac{\log \lfloor 2 a\rfloor !}{\lfloor 2 a\rfloor}+O(1), a \geq 1\end{array}\right.$.

When $D=b n^{2}, 0<b \leq 1 / 2, r_{\tau}^{\mathrm{l}}(D) \leq r(D) \leq \overline{r_{\tau}^{\mathrm{l}}(D)}$, where

$$
\begin{aligned}
& \underline{r_{\tau}^{1}(D)}=\max \left\{0, n \log 1 /\left(2 b e^{2}\right)\right\}, \\
& \overline{r_{\tau}^{\mathrm{l}}(D)}=n \log \lceil 1 /(2 b)\rceil+O(\log n) .
\end{aligned}
$$

Below we first show the achievability of Theorem 6 and Theorem 7 by describing the basic building block of explicit constructions in Section IV-A, where we quantize permutations by sorting. Then for $0<\delta \leq 1$, we analyze the rate-distortion trade-off for the moderate distortion regime $D=\Theta\left(n^{1+\delta}\right)$, small distortion regime $D=\Theta\left(n^{\delta}\right)$ and large distortion regime $D=\Theta\left(n^{2}\right)$ in Section IV-B, Section IV-C and Section IV-D respectively, which corresponds to $\lim _{n} R\left(D_{n}\right) \in[0,1), \lim _{n} R\left(D_{n}\right)=1$, and $\lim _{n} R\left(D_{n}\right)=0$ respectively. Finally, we present converse results in Section IV-E.

\section{A. Quantization by sorting subsequences}

In this section we describe the basic building block for lossy source coding in permutation space $\mathcal{X}\left(\mathcal{S}_{n}, d_{\tau}\right)$ : sorting the subsequences of a given permutation $\sigma \in \mathcal{S}_{n}$. This operation reduces the number of possible permutations and thus the code rate, but introduces distortion. By choosing the proper configuration, we can achieve the corresponding rate-distortion function.

More specifically, we consider the following code construction with parameter $m, k \in \mathbb{Z}^{+}$such that $2 \leq m \leq n$ and $k m \leq n: \mathcal{C}(k, m, n) \triangleq\left\{\pi: \pi=f(\sigma), \sigma \in \mathcal{S}_{n}\right\}$, where $\pi=f(\sigma)$ satisfies

$$
\begin{aligned}
& \pi[i m+1:(i+1) m] \\
& \quad=\operatorname{sort}(\sigma[i m+1:(i+1) m]), 0 \leq i \leq k, \\
& \pi(j)=\sigma(j), j>k m,
\end{aligned}
$$

and $\pi[a: b]$ is a shorthand notation for the vector $[\pi(a), \pi(a+1), \ldots, \pi(b)]$. Then $|\mathcal{C}(k, m, n)|=n ! /\left(m !^{k}\right)$, and we define the $(\log )$ size reduction as

$$
\begin{aligned}
\Delta(k, m) & \triangleq \log \frac{n !}{|\mathcal{C}(k, m, n)|}=k \log m ! \\
& \stackrel{(a)}{=} k\left[m \log m-m \log e+\frac{1}{2} \log m+O\left(\frac{1}{m}\right)\right],
\end{aligned}
$$


where (a) follows from Stirling's approximation in (4). Therefore,

$\Delta(k, m)=k\left[m \log m-m \log e+\frac{1}{2} \log m+O\left(\frac{1}{m}\right)\right]$.

In addition, the maximal distortion $D(k, m)$ of this codebook satisfies $D(k, m) \leq k m(m-1) / 2 \leq k m^{2} / 2$.

Remark 4. Due to the close relationship between Kendall tau distance and $\ell_{1}$ distance shown in (2), there exists an equivalent construction via the inverse permutation $\sigma^{-1}$ of a permutation $\sigma \in \mathcal{S}_{n}$ :

1) Construct a vector $a(\sigma)$ such that for $1 \leq i \leq k$, $a(i)=j$ if $\sigma^{-1}(i) \in[(j-1) m+1, j m], 1 \leq j \leq k$.

Then a contains exactly $m$ values of integers $j$.

2) Form a permutation $\pi^{\prime}$ by replacing the length-m subsequence of a that corresponds to value $j$ by vector $[(j-1) m+1,(j-1) m+2, \ldots, j m]$.

It is not hard to see that the set of $\left\{\pi^{\prime-1}\right\}$ forms a codebook with the same size with distortion upper bounded by $\mathrm{km}^{2} / 2$.

B. Construction for moderate distortion: $D=$ $\Theta\left(n^{1+\delta}\right), 0<\delta<1$

Let $m \triangleq 2\lceil(D-n) / n\rceil=\Theta\left(n^{\delta}\right)$ and set $k \in \mathbb{Z}^{+}$such that $n=k m-q$, where $q \in \mathbb{Z}^{+}$and $0 \leq q \leq m-1$.

1) When $q=0$ : In this case, $n=k m$, and we construct the codebook $\mathcal{C}(k, m, n)$. Then the codebook has distortion

$$
D_{n} \leq k \frac{m^{2}}{2}=k m \frac{m}{2}=n\lceil(D-n) / n\rceil \leq D .
$$

2) When $q>0$ : In this case, we map each $\sigma \in \mathcal{S}_{n}$ to $\sigma^{\prime} \in \mathcal{S}_{n^{\prime}}$ where $n^{\prime}=k m$ and $\sigma^{\prime}(n+i)=n+i$, where $i=$ $1,2, \ldots, q$. Now we can apply the mapping for $q=0$ on $\mathcal{S}_{n^{\prime}}$, for each $\pi^{\prime} \in \mathcal{S}_{n^{\prime}}$, we map it back to $\pi \in \mathcal{S}_{n}$ by truncating the last $q$ coordinates of $\pi^{\prime}$, which are guaranteed to be $n+1, n+2, \cdots, n+q$ by the sorting operation. Furthermore, it is not hard to verify that this achieves the same $M_{n}$ and $D_{n}$ as in the case $q=0$.

Therefore, for any $q$,

$$
\Delta(k, m)=k[m \log m+O(m)]=\delta n \log n+O(n),
$$

and thus $\log M_{n}=\log n !-\Delta(k, m)=(1-\delta) n \log n+$ $O(n)$. Finally, the achievable rate is less than

$$
\lim _{n \rightarrow \infty} \frac{\log M_{n}}{\log n !}=\lim _{n \rightarrow \infty} \frac{(1-\delta) n \log n+O(n)}{\log n !}=1-\delta .
$$

C. Construction for small distortion: $D=a n^{\delta}, 0<\delta \leq 1$

1) When $a \geq 1$ : Let $m=\lfloor 2 a\rfloor$ and $k=\left\lfloor n^{\delta} / m\right\rfloor$, then $\Delta(k, m)=k \log m$ !

$$
\geq\left(n^{\delta} / m-1\right) \log m !=\frac{\log \lfloor 2 a\rfloor !}{\lfloor 2 a\rfloor} n^{\delta}+O(1) .
$$

And the maximal distortion satisfies

$$
D(k, m) \leq k m^{2} / 2 \leq \frac{n^{\delta} m}{2} \leq a n^{\delta}=D .
$$

2) When $0<a<1$ : Let $m=2$ and $k=\lfloor D / 2\rfloor$, then $\Delta(k, m)=k \log m !=\left\lfloor\frac{D}{2}\right\rfloor \log 2=\frac{a \log 2}{2} n^{\delta}+O(1)$.

And the maximal distortion is $D(k, m) \leq k m^{2} / 2 \leq D$.
D. Construction for large distortion: $D=b n(n-1), 0<$ $b \leq 1 / 2$

Let $k=\lceil 1 /(2 b)\rceil$ and $m=\lfloor n / k\rfloor$, then

$\Delta(k, m)=k \log m ! \geq k \log (n / k-1) !$

$$
\begin{aligned}
& \geq k[n / k \log (n / k)-n / k \log e+O(\log n)] \\
& =n \log n-n \log e-n \log \lceil 1 /(2 b)\rceil+O(\log n) .
\end{aligned}
$$

Hence $r(D)=\log n !-\Delta(k, m) \leq \log \lceil 1 /(2 b)\rceil+$ $O(\log n)$. And the maximal distortion satisfies

$$
D(k, m) \leq k m^{2} / 2 \leq n^{2} /(2 k) \leq n^{2} /(1 / b)=b n^{2} .
$$

\section{E. Converse}

Given the maximum size of the $D$-balls in permutation spaces, the converse proof is straightforward.

Proof for the converse of Theorem 6: For any D, $|\mathcal{C}| N_{\tau}(D) \geq n$ !, and according to Lemma 4,

$\log |\mathcal{C}| \geq \log n !-\log N_{\tau}(D) \geq \log n !-\delta n \log n-O(n)$.

Therefore, $\lim _{n \rightarrow \infty} \log |\mathcal{C}| /(\log n !) \geq 1-\delta$.

Proof for Theorem 7: The upper bounds are based on constructions Section IV-C and Section IV-D, while the lower bounds follow from Lemmas 3 to 5 .

\section{Permutation space with $\ell_{1}$ Distance of the INVERSION VECTORS}

Parallel to the development in Section IV, in this section we derive the rate-distortion function $R(D)$ for the permutation space $\mathcal{X}\left(\mathcal{S}_{n}, d_{\mathbf{x}, \ell_{1}}\right)$ and the higher order term $r(D)$ in the small and large distortion regimes, with the results summarized in Theorem 8 and Theorem 9 .

Theorem 8. In the permutation space $\mathcal{X}\left(\mathcal{S}_{n}, d_{\mathbf{x}, \ell_{1}}\right)$, where $d_{\mathbf{x}, \ell_{1}}(\cdot, \cdot)$ is defined in Section II,

$$
R(D)=\left\{\begin{array}{ll}
1 & \text { if } D=O(n) \\
1-\delta & \text { if } D=\Theta\left(n^{1+\delta}\right), \quad 0<\delta \leq 1
\end{array} .\right.
$$

Theorem 9. In the permutation space $\mathcal{X}\left(\mathcal{S}_{n}, d_{\mathbf{x}, \ell_{1}}\right)$, when $D=a n^{\delta}, 0<\delta \leq 1$,

$$
\underline{r_{\mathbf{x}, \ell_{1}}^{\mathrm{s}}(D)} \leq r(D) \leq \overline{r_{\mathbf{x}, \ell_{1}}^{\mathrm{s}}(D)},
$$

where $r_{\mathbf{x}, \ell_{1}}^{\mathrm{s}}(D)=\underline{r_{\tau}^{\mathrm{s}}(D)}-n^{\delta} \log 2(c f .(14))$ and

$$
\overline{r_{\mathbf{x}, \ell_{1}}(D)}=\left\{\begin{array}{ll}
-\left\lfloor n^{\delta}\right\rfloor \log (2 a-1) & a>1 \\
-\left\lceil a n^{\delta}\right\rceil \log 3 & 0<a \leq 1
\end{array} .\right.
$$

When $D=b n^{2}, 0<b \leq 1 / 2$,

$$
\underline{r_{\mathbf{x}, \ell_{1}}^{\mathrm{l}}(D)} \leq r(D) \leq \overline{r_{\mathbf{x}, \ell_{1}}^{\mathrm{l}}(D)},
$$

where $r_{\mathbf{x}, \ell_{1}}^{\mathrm{l}}(D)=\underline{r_{\tau}^{\mathrm{l}}(D)}\left(c f\right.$. (15)) and $\overline{r_{\mathbf{x}, \ell_{1}}^{\mathrm{l}}(D)}=$ $n \log \lceil\overline{1 /(4 b)\rceil+} O(1)$.

Below we first show a variety of constructions for the different regimes of interest in Sections V-A to V-C, where the quantization for a permutation vector is simply componentwise scalar quantization of its inversion vector. This suffices due to the product structure of inversion vector space $\mathcal{G}_{n}$. Note that to quantize $k$ points in $[0: k-1]$, where $k=$ $2, \cdots, n$ uniformly with $m$ points, the maximal distortion 
is $D_{k}=\lceil(k / m-1) / 2\rceil$. Conversely, to achieve distortion $D_{k}$, we need $m \geq\left\lceil k /\left(2 D_{k}+1\right)\right\rceil$ points. The converse (lower bounds) proofs for Theorem 8 and Theorem 9 is similar to the converse proofs Section IV and hence omitted.

A. Construction for moderate distortion: $D=$ $\Theta\left(n^{1+\delta}\right), 0<\delta<1$

Let $D_{k}=(k D) /\left((n+2)^{2}\right)$. Then we need

$$
m_{k}=\left\lceil k /\left(2 D_{k}+1\right)\right\rceil \leq\left\lceil\frac{k(n+2)^{2}}{2 k D}\right\rceil=\left\lceil\frac{(n+2)^{2}}{2 D}\right\rceil \text {. }
$$

The overall distortion $D_{n}$ satisfies

$$
D_{n}=\sum_{k=2}^{n} D_{k}=\frac{(n-1)(n+2) D}{(n+2)^{2}} \leq D,
$$

and the codebook size $M_{n}$ satisfies

$$
\begin{aligned}
\log M_{n} & =\sum_{k=2}^{n} \log m_{k} \leq n \log \left\lceil\frac{(n+2)^{2}}{2 D}\right\rceil \\
& =(1-\delta) n \log n+O(n) .
\end{aligned}
$$

Therefore, $R(D) \leq \lim _{n \rightarrow \infty} \log M_{n} /(\log n !)=1-\delta$.

B. Construction for small distortion: $D=a n^{\delta}, 0<\delta \leq 1$

$$
\begin{aligned}
& \text { 1) } a>1 \text { : Let } \\
& m_{k}=\left\{\begin{array}{ll}
k & k \leq n-\left\lfloor n^{\delta}\right\rfloor \\
\lceil k /(2 a-1)\rceil & k>n-\left\lfloor n^{\delta}\right\rfloor
\end{array} \quad k=2, \ldots, n\right.
\end{aligned}
$$

then the distortion $D_{k}$ for each coordinate $k$ satisfies $k=$ $2,3, \ldots, n$.

$$
D_{k} \leq\left\{\begin{array}{ll}
a & k \leq\left\lceil n^{\delta}\right\rceil \\
0 & k>\left\lceil n^{\delta}\right\rceil
\end{array}, k=2,3, \ldots, n,\right.
$$

and hence $D_{n}=\sum_{k=2}^{n} D_{k}=\left(\left\lfloor n^{\delta}\right\rfloor\right) a \leq D$. In addition, the codebook size

$$
M_{n}=\prod_{k=2}^{n} m_{k} \leq(1 /(2 a-1))^{\left\lfloor n^{\delta}\right\rfloor} \prod_{k=2}^{n} k .
$$

Therefore, $\log M_{n} \leq \log n !-\left\lfloor n^{\delta}\right\rfloor \log (2 a-1)+O(\log n)$.

2) $a \leq 1$ : Since $a \leq 1, D=a n^{\delta} \leq n$.

$$
m_{k}=\left\{\begin{array}{ll}
\lceil k / 3\rceil & k<\lceil D\rceil \\
k & k \geq\lceil D\rceil
\end{array}, \quad k=2, \ldots, n\right.
$$

and apply uniform quantization on the coordinate $k$ of the inversion vector with $m_{k}$ points, Then the distortion $D_{k}$ for each coordinate $k$ satisfies

$$
D_{k} \leq\left\{\begin{array}{ll}
1 & k<\lceil D\rceil \\
0 & k \geq\lceil D\rceil
\end{array}, k=2,3, \ldots, n,\right.
$$

and hence $D_{n}=\sum_{k=2}^{n} D_{k}=\lceil D\rceil-1 \leq D$. In addition, the codebook size

$$
\begin{aligned}
M_{n} & =\prod_{k=2}^{n} m_{k} \leq \prod_{k=2}^{\lceil D\rceil-1}(k+3) / 3 \prod_{k=\lceil D\rceil}^{n} k \\
& =\frac{1}{3^{\lceil D\rceil-1}}\lceil D\rceil(\lceil D\rceil+1)(\lceil D\rceil+2) \prod_{k=5}^{n-1} k .
\end{aligned}
$$

Therefore, $\log M_{n} \leq \log n !-\left\lceil a n^{\delta}\right\rceil \log 3+O(\log n)$.
C. Construction for large distortion: $D=b n(n-1), 0<$ $b \leq 1 / 2$

Let $m_{k}=\lceil k /(4 b(k-1)+1)\rceil, \quad k=2, \ldots, n$. The distortion $D_{k}$ for each coordinate $k$ satisfies

$$
D_{k}=\left\lceil\frac{1}{2}\left(\frac{k}{m}-1\right)\right\rceil \leq\lceil 2 b(k-1)\rceil, k=2,3, \ldots, n,
$$

and hence $D_{n}=\sum_{k=2}^{n} D_{k} \leq \sum_{k=2}^{n} 2 b(k-1)+1 \leq$ $(b+1 / n) n(n-1)$. In addition, the codebook size

$$
M_{n}=\prod_{k=2}^{n} m_{k} \leq \prod_{k=2}^{n}\left\lceil\frac{k-1}{4 b(k-1)}\right\rceil \leq\left\lceil\frac{1}{4 b}\right\rceil^{n-1} .
$$

Therefore, $\log M_{n} \leq n \log \lceil 1 /(4 b)\rceil+O(1)$.

\section{CONCLUding REMARKS}

This work can be extended in a few directions. First, tightening the bounds in Section III will lead to better converse results for Sections IV and V. Second, depending on the applications, it may be useful to consider other distortion metrics such as the Chebyshev distance. Furthermore, with aforementioned relation to approximate sorting (cf. Section I), it is of interest to develop approximate sorting algorithms that achieve near-optimal performance in the small distortion regime, in terms of the lower bound specified in Theorem 7 . These directions are currently under investigation.

\section{REFERENCES}

[1] I. Blake, G. Cohen, and M. Deza, "Coding with permutations," Information and Control, vol. 43, no. 1, pp. 1-19, 1979.

[2] C. Colbourn, T. Klove, and A. Ling, "Permutation arrays for powerline communication and mutually orthogonal latin squares," IEEE Trans. Inf. Theory, vol. 50, no. 6, pp. 1289-1291, 2004.

[3] T. Klve, T. Lin, S. Tsai, and W. Tzeng, "Permutation arrays under the Chebyshev distance," IEEE Trans. Inf. Theory, vol. 56, no. 6, pp. 2611-2617, 2010.

[4] A. Barg and A. Mazumdar, "Codes in permutations and error correction for rank modulation," IEEE Trans. Inf. Theory, vol. 56, no. 7, pp. 3158-3165, 2010.

[5] J. Barbay and G. Navarro, "Compressed representations of permutations, and applications," in Proc. 26th International Symposium on Theoretical Aspects of Computer Science STACS 2009, 2009, pp. 111-122.

[6] - "On compressing permutations and adaptive sorting," arXiv:1108.4408, Aug. 2011. [Online]. Available: http://arxiv.org/abs/1108.4408

[7] J. Barbay, J. Fischer, and G. Navarro, "LRM-Trees: compressed indices, adaptive sorting, and compressed permutations," Theoretical Computer Science, vol. 459, no. 0, pp. 26-41, 2012.

[8] J. Giesen, E. Schuberth, and M. Stojakovi, "Approximate sorting," in LATIN 2006: Theoretical Informatics. Springer, Jan. 2006, no. 3887, pp. 524-531.

[9] P. Diaconis and R. L. Graham, "Spearman's footrule as a measure of disarray," Journal of the Royal Statistical Society. Series B (Methodological), vol. 39, no. 2, pp. 262-268, 1977.

[10] D. E. Knuth, Art of Computer Programming, Volume 3: Sorting and Searching, 2nd ed. Addison-Wesley Professional, May 1998.

[11] A. Mazumdar, A. Barg, and G. Zemor, "Constructions of rank modulation codes," IEEE Trans. Inf. Theory, vol. 59, no. 2, pp. 10181029, 2013.

[12] R. Shreevatsa, "The On-Line Encyclopedia of Integer Sequences," 2013. [Online]. Available: http://oeis.org/A161169

[13] R. P. Stanley, Enumerative Combinatorics, Vol. 1. Cambridge University Press, Apr. 1997.

[14] G. Louchard, H. Prodinger, and P. O. Wits, "The number of inversions in permutations: a saddle point approach," Journal of Integer Sequences, vol. 6, no. 2, p. 3, 2003. 